\title{
Protein standardization V: value transfer. A practical protocol for the assignment of serum protein values from a Reference Material to a Target Material
}

\author{
International Federation of Clinical Chemistry \\ and Laboratory Medicine (IFCC) ${ }^{1}$
}

IFCC Scientific Division ${ }^{2}$

\author{
Søren Blirup-Jensen ${ }^{1, *}$, A. Myron Johnson ${ }^{2}$ \\ and Marianne Larsen ${ }^{3}$ on behalf of the IFCC \\ Committee on Plasma Proteins \\ ${ }^{1}$ Department of Clinical Chemistry, University \\ Hospital, Lund, Sweden \\ ${ }^{2}$ Departments of Pediatrics and of Obstetrics and \\ Gynecology, University of North Carolina School of \\ Medicine, Chapel Hill, NC, USA \\ ${ }^{3}$ Clinical Immunochemistry, Dako A/S, Copenhagen, \\ Denmark
}

\begin{abstract}
We present a practical protocol for the assignment of values to serum proteins in a Target Material using a Reference Material. This protocol is based on the model of Direct Value Transfer between serum matrices and is intended to improve the value assignment of commercial calibrators using the Reference Material CRM 470 (now labeled ERM-DA 470) or similar
\end{abstract}

\footnotetext{
1) The exclusive (C) for all languages and countries is vested in the International Federation of Clinical Chemistry and Laboratory Medicine.

2) IFCC Sections printed in J. Clin. Chem. Clin. Biochem. are listed in the Cumulative Index, which appeared in connection with the contents of this journal in Volume 27, 1989 and since 1991 have been printed in (Eur.) J. Clin. Chem. Clin. Biochem.

IFCC 1991/1 Vol. 29, 435-457

IFCC 1991/2 Vol. 29, 531-535

IFCC 1991/3 Vol. 29, 577-586

IFCC 1991/4 Vol. 29, 767-772

IFCC 1992/1 Vol. 30, 901-905

IFCC 1994/1 Vol. 32, 639-655

IFCC 1995/1 Vol. 33, 247-253

IFCC 1995/2 Vol. 33, 399-404

IFCC 1995/3 Vol. 33, 623-625

IFCC 1995/4 Vol. 33, 627-636

IFCC 1995/5 Vol. 33, 637-660

IFCC 1997/1 Vol. 35, 317-344

IFCC 1997/2 Vol. 35, 345-349

IFCC 1997/3 Vol. 35, 805-831

IFCC 1997/4 Vol. 35, 833-843

For IFCC sections printed in Clin. Chem. Lab. Med. since 1998, please visit the link http://degruyter.com/journals/ extenza, where they are freely accessible.

*Corresponding author: S. Blirup-Jensen, DVM, PhD,

Department of Clinical Chemistry, Lund University Hospital, 22185 Lund, Sweden

Phone: +46-46-173465, Fax: +46-46-130064,

E-mail: Soren.Blirup@med.lu.se

Received for publication June 25, 2008;

previously published online August 29, 2008
}

reference materials. The procedure describes the general as well as the practical principles involved in the value assignment (with examples). The practical transfer protocol is based on multiple assays of 6 dilutions of the Reference Material and 6 dilutions of the Target Material. The transfer protocol requires several measurements a day repeated on several days, an important prerequisite being that all reconstitutions and dilutions are controlled by weighing thus reducing uncertainty in the transfer. In open systems that allow the use of the Reference Material as calibrator and the Target Material as samples, the proportionality of the two materials (the presence or absence of matrix effects) can now be directly assessed by evaluating a single regression plot. If no matrix effects are found, the regression line will pass through zero with a slope equal to the ratio of the concentrations of the two materials. In closed systems, the dedicated commercial calibrator has to be used as such; the Reference Material and the Target Material are now assayed as samples against this calibrator. Two regression plots are therefore obtained; if no matrix effects are present among the two materials and the calibrator, both the Reference and Target Materials will show zero intercepts, and the ratio of the two slopes will equal the ratio of the concentrations.

Clin Chem Lab Med 2008;46:1470-9.

Keywords: protein standardization; transfer protocol; value assignment; value transfer.

Abbreviations: $\mathbf{C}_{\mathbf{R}}^{\prime}=$ Stated analyte concentration in the Reference Material; $\mathbf{C}_{\mathbf{R}}=$ Actual analyte concentration in the Reference Material after reconstitution; $\mathbf{C}_{\mathrm{T}}=$ Unknown analyte concentration in the Target Material; $\mathbf{F}_{\mathrm{R}}(\mathbf{1 - 6})=$ Relative concentration factors 1-6 of the Reference Material obtained by interpolation; $\mathbf{F}_{\mathrm{T}} \mathbf{1}=$ Predilution factor $\mathbf{1}$ for the Target Material; $\mathbf{F}_{\mathrm{T}} \mathbf{2}=$ Dilution factor $\mathbf{2}$ for the Target Material; $\mathbf{M}_{\mathbf{X}}=$ Mass of $x$ (where $x$ can be: reconstituent intended, water, Reference Material, Dilution buffer or Target Material); $\mathbf{R}=$ Correction factor for the Reference Material after reconstitution; $\mathbf{T}=$ Correction factor for the Target Material after reconstitution; $\mathbf{U}_{\mathbf{Y}}=$ Uncertainty of $\mathrm{Y}$ (where $\mathrm{Y}$ can be: Total, Reference Material or Transfer procedure); $\boldsymbol{\alpha}=$ Intercept of the linear regression line; $\boldsymbol{\beta}=$ Slope of the linear regression line.

\section{Introduction}

When the international Reference Preparation for Human Serum Proteins (CRM 470) was prepared (1), procedures and protocols were developed for the transfer of values to this material (2). This new approach for the assignment of values to serum pro- 
teins in a Target Material using a Reference Preparation was developed and published by Blirup-Jensen, Johnson and Larsen (3).

The introduction of the Certified Reference Material (CRM 470, now referred to as ERM-DA 470) has definitely improved the worldwide results of quantitative serum protein determinations as seen from the various national quality assurance programs (4-7).

However, differences as large as $15 \%$ among manufacturers' assays persist for some proteins. Several factors may contribute to this, including (among others) different antiserum reactivities, assay methods, genetically determined structural variation in the proteins themselves, inadequate methods of value transfer, and matrix differences among the materials. In a given case, any or all of these may be operable, and the results may be additive. The first three of these are difficult if not impossible to control.

In our first publication (3), we focused on a general discussion of the value transfer principles and presented the necessary mathematical equations. However, following the development we have realized the necessity - also encouraged by many users - to produce a more practical (easy to follow) protocol for the value transfer. Therefore, in an attempt to aid manufacturers in transferring values accurately and thereby to reduce the among-manufacturer bias in serum protein assays, the authors have been encouraged to prepare a protocol with simple mathematical calculations and some practical recommendations to be used when values are to be transferred from certified reference materials to manufacturers' working calibrators (master calibrators) and then to their commercial calibrators and controls.

The methods used today for serum protein assays are based on immunochemical principles utilizing the specific reaction between an antigen and its corresponding antibody.

Common to all quantitative methods is the basic principle of calibrating the assay, i.e., recording the signals from different dilutions of a calibrator with known concentration of the actual protein. Once the instrument has been calibrated, unknown patient samples can be assayed and quantified by interpolation on the established standard curve.

As part of the daily routine, the clinical laboratory may perform a considerable number of specific quantitative protein determinations. In order to do this, reliable assays are required. During the last decade, the original gel precipitation techniques have increasingly been replaced by fast and automated optical detection systems, which take advantage of the fast and specific reaction between an antigen and the corresponding antibody in a liquid phase. Most of these systems are based on immunoturbidimetry and immunonephelometry, measuring the light absorbed or scattered by the antigen-antibody complexes formed in free solution. Earlier studies (8) have shown that, due to the fast reaction between the antigen and the antibody, optimal programming of the instrument is a very important factor for obtaining reliable and reproducible results.

The different reagents also play an important role. Slight variations in the specificity and titer of the anti- body may cause erroneous results. Likewise, the composition of the reaction buffer, the $\mathrm{pH}$ and the polymer concentration may influence the accuracy of the final result. This has led to the prerequisite of Method Standardization (8).

Provided that the instrument is programmed correctly and the reagents used are of sufficient quality, the concentration in a patient sample will be expressed in relation to the value of the calibrator. It is therefore of the highest importance that the calibrator be value assigned correctly.

\section{General principles}

The goal of the value transfer is to assign concentrations to selected proteins in a Target Material using an International Reference Preparation. In order to do this, Transfer Methods must be selected and Transfer Protocols developed.

Reference Material $\stackrel{\text { Transfer Method }}{\text { Transfer Protocol }}$ Target Material

The Reference Material (earlier also referred to as the Reference Preparation) is defined as the protein preparation with known concentration values (e.g., assigned using a consensus procedure). In this example of value transfer, the Reference Material CRM 470 (now referred to as ERM-DA 470) is used. The preparation and value assignment of this material were documented in 1993 (1). Since the original Reference Material is gradually coming to an end, a replacement material with similar values is currently being made by the Institute for Reference Materials and Methods (IRMM), Geel, Belgium, and should be available during 2008.

The Target Material is defined as the serum protein matrix material (e.g., a manufacturer's calibrator) with unknown concentration values.

The Transfer Method should be a well established and a recognized routine method, such as turbidimetry, nephelometry or single radial immunodiffusion.

However, slight variations in the assay conditions, in the programming of the instruments or in the reagents may lead to different results. This has led to the prerequisite of Method Standardization (8).

To minimize all of these factors, an accurate Transfer Protocol with detailed instructions, dilution schemes, parameter settings, etc., should also be used.

Finally data reduction and proper statistical evaluation are required for certification of the values obtained.

The practical protocol for value transfer outlined in this paper is an attempt to describe in detail how a value assignment can be conducted in order to minimize the variability and differences on the transfer of values to manufacturers' calibration and control materials for serum proteins.

To illustrate the various aspects of the practical protocol, a real example is presented with a detailed description of each step of the procedure, in which the concentration value of human $\lg G$ is value assigned to a Target Material (a human serum 
matrixed material) from a Reference Material (CRM 470 or now ERM-DA 470).

\section{General procedure for the direct value assignment (the Transfer Protocol)}

The Transfer Protocol is based on multiple-point value assignment obtained by several measurements a day repeated on several days.

All volumes for reconstitution and dilutions must be controlled by weight using a balance with $0.0001 \mathrm{~g}$ precision.

Reference Material: 6 different dilutions used as standards for the calibration curve and covering the actual measuring range.

Target Material: $\quad 6$ different dilutions distributed within the measuring range and placed as samples in 2 positions.

Control: $\quad 1$ special dilution of the Reference Material in the higher end of the measuring range and placed as a sample in 2 positions.

Each day, a new set of dilutions is prepared and 3 calibrations including determinations of samples and controls are carried out.

This procedure for 1 day is repeated for 4 days thus giving:

Calibration curves: 6 different dilutions of the Reference Material used as standards repeated 3 times each day over 4 days $=6 \times 3 \times 4=72$ determinations.

Samples: 6 different dilutions of the Target Material analyzed in duplicate 3 times a day in 4 days $=$ $6 \times 2 \times 3 \times 4=144$

determinations.

Controls:

1 special dilution of the Reference Material analyzed in duplicate 3 times a day in 4 days $=$ $2 \times 3 \times 4=24$ determinations.

\section{Materials}

The value assignment can be performed in any optimized and validated immunochemical system. It is highly recommended to use the appropriate setup for each specific instrument. In the example described in this paper, the following reagents and instruments were used:

- Reference Material: CRM 470 91/06 19 (IRMM, Geel, Belgium).

- Target Material: Human Serum Protein Calibrator X 908 (DAKO A/S, Copenhagen, Denmark).
- Reaction Buffer: S 2007 (DAKO A/S).

- Dilution Buffer: S 2005 (DAKO A/S).

- Saline Solution: 0.1 M NaCl, 15 mM Na-azide.

- Distilled or dematerialized water.

- Antibody: Rabbit anti-human IgG O 0331 (DAKO $\mathrm{A} / \mathrm{S})$.

- Turbidimeter: Cobas Fara II (F. Hoffmann-La Roche Ltd., Basel, Switzerland).

- Balance: Sartorius, MC 410 S, Sartorius, Germany.

\section{Practical procedure for the direct value assignment}

Setup for 1 day For the following example, Day \#4 from a real value transfer of human IgG has been chosen.

Registration:

1. The following are documented on a special registration form:

a. The specific serum protein to be value assigned - in this example human $\lg G$.

b. The date for the first run.

c. The operator.

d. The instrument (Name, Producer, Model and Lab. Instr. No.).

e. The Reference Material (Name, Producer, Code No., Lot. No. and the concentration of the chosen protein plus the uncertainty), e.g., ERM-DA 470 (CRM 470), IRMM, Lot no. 91/06 19, IgG conc. $9.68 \mathrm{~g} / \mathrm{L}$, uncertainty $0.10 \mathrm{~g} / \mathrm{L}$.

f. The Target Material (Name, Producer, Code No., Lot. No.).

g. Reaction Buffer (Name, Producer, Code No., Lot. No.).

h. Antibody (Name, Producer, Code No., Lot. No.).

i. Dilution Buffer for Standards (Name, Producer, Code No., Lot. No.).

j. Dilution Buffer for Samples (Name, Producer, Code No., Lot. No.).

k. Saline for Predilution (Name, Producer, Code No., Lot. No.).

Pre-preparation:

2. Frozen material is thawed at $37^{\circ} \mathrm{C}$ the afternoon before the day of the measurements. After thawing, it is left next to the balance (at room temperature) overnight.

3. Freeze-dried material is reconstituted the afternoon before the day of the measurements and according to the product insert. If no product insert is available, this general recommendation may be used:

a. Leave the vial at room temperature for at least $2 \mathrm{~h}$ before beginning.

b. Tap the vial gently on the surface of the table assuring that all material has settled onto the bottom of the vial.

c. Remove the metal seal or screw cap.

d. Weigh the vial together with the rubber stopper and record the weight.

e. Lift the rubber stopper with care until air is allowed to enter and the groove in the rubber stopper becomes accessible. 
f. With a precision pipette add $1000 \mu \mathrm{L}$ (or other specified volume) of distilled water and press the rubber stopper back into place.

g. Reweigh the vial and record the weight.

h. Leave the vial at room temperature for approximately $1 \mathrm{~h}$.

i. Now carefully invert it several times (do not shake it!) during the following hour.

j. Leave the vial next to the balance overnight.

Since the Reference Preparation (CRM 470) is freeze-dried, the material is reconstituted as described above. The actual weighing results are illustrated in Table 1.

If the IgG value from the package insert is stated to be $9.68 \mathrm{~g} / \mathrm{L}\left(\mathbf{C}_{\mathrm{R}}^{\prime}\right)$ when reconstituted with $1.0000 \mathrm{~mL}$ water, the actual value $\left(\mathbf{C}_{R}\right)$ is now:

$\mathrm{C}_{\mathrm{R}}=\frac{\mathbf{C}_{\mathrm{R}}^{\prime} \times \mathbf{M}_{\text {Recon. }}}{\mathbf{M}_{\text {water }}}=\frac{9.68 \times 1.0000}{0.9950}=\mathbf{9 . 7 2 9} \mathrm{g} / \mathrm{L}$

where $\mathbf{M}$ is the mass of the reconstituent intended as well as actual. A correction factor $\mathbf{R}=\mathbf{M}_{\text {Recon }} / \mathbf{M}_{\text {Water }}$ could also be used directly, giving: $\mathbf{C}_{R}=\mathbf{C}_{R}^{\prime} \times \mathbf{R}$.

If the Target Material must be reconstituted, a similar procedure is followed and Table 2 is used. However, in this example the Target Material is already a

Table 1 Reconstitution of Reference Preparation.

\begin{tabular}{ll}
\hline & Reference Preparation \\
\hline Vial + stopper + water, g & 7.6852 \\
Vial + stopper, g & 6.6902 \\
Water, g & 0.9950 \\
Correction factor, R & 1.005025 \\
\hline
\end{tabular}

Table 2 Reconstitution of Target Material.

\begin{tabular}{ll}
\hline & Target Material \\
\hline Vial + stopper + water, g & 0.0000 \\
Vial + stopper, g & 0.0000 \\
Water, g & 0.0000 \\
Correction factor, T & 1.00000 \\
\hline
\end{tabular}

liquid serum, so reconstitution and weighing is not needed.

Calibration standards:

4. 6 different dilutions are prepared from the reconstituted Reference Material. They should serve as standards for the calibration curve and should cover the actual measuring range. If the concentration of the chosen protein is very high, a predilution of the Reference Material may be required before the 6 standards are made. For $\lg$, this is not necessary and the 6 standards are prepared according to Table 3.

An example of the actual weighing results is illustrated in Table 4.

The relative concentrations in \% for Std. 2 and Std. 4 are calculated as:

Rel. conc. (Std. 2) $=\frac{\mathbf{M}_{R} \times 100}{M_{R}+M_{\text {Dil }}}=\frac{0.993 \times 100}{0.0993+0.9025}$ $=\mathbf{9} .9122 \%$

Rel. conc. $($ Std. 4$)=\frac{M_{R} \times \text { Std. 2 }}{M_{R}+M_{\text {Dil }}}=\frac{0.3935 \times 9.9122}{0.3935+0.3500}$ $=5.2461 \%$

To avoid pipetting very small volumes, Std. 4 , Std. 5 and Std. 6 are prepared from Std. 2, Std. 3 and Std. 5 , respectively. In total, $500 \mu \mathrm{L}$ of each of the different dilutions is transferred to a separate calibrator cup and placed on the instrument in 6 consecutive calibrator positions according to the instruction manual for the specific instrument. The relative concentration values in \% are entered into the appropriate calibration software window.

It is preferable to prepare all standards manually by weighing all volumes as described. However, for some instruments this is not possible, because the instrument automatically prepares the 6 different standards (dilutions) from the single calibrator.

Table 3 Human IgG - intended dilution scheme of the Reference Material.

\begin{tabular}{|c|c|c|c|c|c|c|}
\hline & Std. 1 & Std. 2 & Std. 3 & Std. 4 & Std. 5 & Std. 6 \\
\hline Dilution buffer, g & 0.7000 & 0.9000 & 1.2000 & 0.3500 & 0.6000 & 0.5000 \\
\hline Reference Material, g & 0.1000 & 0.1000 & 0.1000 & 0.4000 (Std. 2) & 0.6000 (Std. 3) & 0.4000 (Std. 5) \\
\hline Dilution factor & 0.1250 & 0.1000 & 0.0769 & 0.5333 & 0.5000 & 0.4444 \\
\hline Predilution factor & 1.0000 & 1.0000 & 1.0000 & 0.1000 & 0.0769 & 0.0384 \\
\hline Relative concentration, \% & 12.5000 & 10.0000 & 7.6923 & 5.3333 & 3.8450 & 1.7087 \\
\hline
\end{tabular}

Table 4 Human IgG - actual dilution scheme of the Reference Material.

\begin{tabular}{|c|c|c|c|c|c|c|}
\hline & Std. 1 & Std. 2 & Std. 3 & Std. 4 & Std. 5 & Std. 6 \\
\hline Dilution buffer, g & 0.7025 & 0.9025 & 1.1967 & 0.3500 & 0.6002 & 0.4985 \\
\hline Reference Material, g & 0.0999 & 0.0993 & 0.0997 & 0.3935 (Std. 2) & 0.5935 (Std. 3) & 0.3922 (Std. 5) \\
\hline Dilution factor & 0.1245 & 0.0991 & 0.0769 & 0.5293 & 0.4972 & 0.4403 \\
\hline Predilution factor & 1.0000 & 1.0000 & 1.0000 & 0.0991 & 0.0769 & 0.0382 \\
\hline Relative concentration, $\%$ & 12.4501 & 9.9122 & 7.6905 & 5.2461 & 3.8237 & 1.6837 \\
\hline
\end{tabular}


Table 5 Actual dilution of Control Sample.

\begin{tabular}{ll}
\hline & Reference Material \\
\hline Weight of Reference Material, g & 0.0997 \\
Weight of Dilution Buffer, g & 1.0060 \\
Dilution factor & 0.0902 \\
Relative concentration, \% & 9.0169 \\
\hline
\end{tabular}

Control sample:

5. The Control Sample is made as a special dilution of the Reference Material (Table 5). It serves as a curve control and should therefore be made so it falls within the calibration curve. Weigh, e.g., $0.1000 \mathrm{~g}$ reference serum $+1.000 \mathrm{~g}$ Dilution buffer and mix.

In total, $500 \mu \mathrm{L}$ of the control is transferred to each of two sample cups and placed on the instrument in 2 positions.

Sample preparation:

6. If the Target Material is frozen or freeze-dried, it should be thawed or reconstituted as described under the section Pre-preparation. This could similarly give rise to a Correction Factor $(T)$.

6 different dilutions are prepared from the (reconstituted) Target Material. They should serve as samples and should all be made so their concentrations fall within the calibration curve. A trial run may be

Table 6 Actual predilution of Target Material.

\begin{tabular}{ll}
\hline & Target Material \\
\hline Weight of Target Material, g & 0.3989 \\
Weight of saline, $\mathbf{g}$ & 3.9761 \\
Total Weight, g & 4.3750 \\
Predilution factor, $\mathbf{F}_{\mathbf{T}} \mathbf{1}$ & 0.091177 \\
\hline
\end{tabular}

necessary to establish an approximate concentration of the protein in the Target Material.

For human IgG, a predilution of the Target Material is recommended. The intended predilution is $(1+10=11 \times$ or 0.09090$): 0.4000 \mathrm{~g}$ Target Material + $4.0000 \mathrm{~g}$ dilution buffer. The actual predilution is illustrated in Table 6.

6 different dilutions are now prepared from the prediluted Target Material according to Table 7.

An example of the actual weighing results of the 6 samples is illustrated in Table 8.

The dilution factor $\mathbf{F}_{\mathbf{T}} \mathbf{2}$ for Sample 3 is calculated as:

$$
\mathrm{F}_{\mathrm{T}} 2(3)=\frac{\mathrm{M}_{\mathrm{T}}}{\left(\mathrm{M}_{\mathrm{T}}+\mathrm{M}_{\mathrm{Dil}}\right)}=\frac{0.7918}{0.7918+0.2012}=\mathbf{0 . 7 9 7 4}
$$

The relative concentration in \% for Sample 3 is calculated as:

$$
\begin{aligned}
\text { Rel. conc. (Sample 3) } & =\frac{\mathbf{M}_{\mathrm{T}} \times 100 \times \mathrm{F}_{\mathrm{T}} \mathbf{1}}{\left(\mathrm{M}_{\mathrm{T}}+\mathbf{M}_{\mathrm{Dil}}\right)} \\
& =\frac{0.7918 \times 100 \times 0.09118}{(0.7918+0.2012)} \\
& =\mathbf{7 . 2 7 0 3 \%}
\end{aligned}
$$

The relative concentration in \% of the Target Material is useful to verify that the dilutions fall within the measuring range of the calibration curve (prepared from the Reference Material). However, for further calculation the dilution factors $\mathbf{F}_{\mathrm{T}} \mathbf{2}$ (1-6) are used as $X$-values representing the exact concentrations of IgG in the samples (prepared from the Target Material).

$500 \mu \mathrm{L}$ of each of the different dilutions is transferred to each of two sample cups and placed on the instrument as samples in $12(2 \times 6)$ consecutive positions according to the instruction manual for the specific instrument.

\section{Measurements:}

\begin{tabular}{|c|c|c|c|c|c|c|}
\hline & Sample 1 & Sample 2 & Sample 3 & Sample 4 & Sample 5 & Sample 6 \\
\hline Dilution buffer, g & 0 & 0.1000 & 0.2000 & 0.4000 & 0.6000 & 0.7500 \\
\hline Target material, g & 1.0000 & 0.9000 & 0.8000 & 0.6000 & 0.4000 & 0.2500 \\
\hline Total Weight, g & 1.0000 & 1.0000 & 1.0000 & 1.0000 & 1.0000 & 1.0000 \\
\hline Dilution factor, $\mathbf{F}_{\mathrm{T}} \mathbf{2}$ & 1.0000 & 0.9000 & 0.8000 & 0.6000 & 0.4000 & 0.2500 \\
\hline Predilution factor, $\mathbf{F}_{\mathbf{T}} \mathbf{1}$ & 0.09090 & 0.09090 & 0.09090 & 0.09090 & 0.09090 & 0.09090 \\
\hline Relative concentration, $\%$ & 9.0909 & 8.1818 & 7.2727 & 5.4545 & 3.6363 & 2.2727 \\
\hline
\end{tabular}

7. Reagents and buffers are loaded onto the instrument, which is programmed as required to give

\begin{tabular}{|c|c|c|c|c|c|c|}
\hline & Sample 1 & Sample 2 & Sample 3 & Sample 4 & Sample 5 & Sample 6 \\
\hline Dilution buffer, g & 0.0000 & 0.1005 & 0.2012 & 0.4063 & 0.6025 & 0.7513 \\
\hline Target material, g & 1.0000 & 0.8885 & 0.7918 & 0.5897 & 0.3979 & 0.2465 \\
\hline Total weight, g & 1.0000 & 0.9890 & 0.9930 & 0.9960 & 1.0004 & 0.9978 \\
\hline Dilution factor, $\mathbf{F}_{\mathrm{T}} \mathbf{2}$ & 1.0000 & 0.8984 & 0.7974 & 0.5921 & 0.3977 & 0.2470 \\
\hline Predilution factor, $\mathbf{F}_{\mathrm{T}} \mathbf{1}$ & 0.091177 & 0.091177 & 0.091177 & 0.091177 & 0.091177 & 0.091177 \\
\hline Relative concentration, $\%$ & 9.1177 & 8.1912 & 7.2703 & 5.3983 & 3.6265 & 2.2525 \\
\hline
\end{tabular}

Table 7 Human IgG - intended sample dilution scheme of the prediluted Target Material.

Table 8 Human IgG - actual sample dilution scheme of the prediluted Target Material. 
the best possible determination of the specific protein (see refs. 3, 8).

8. The instrument is started, and 3 complete runs (calibrations and determinations of samples and controls) are carried out. This corresponds to 1 day in the value assignment procedure. The following data are printed: Parameter settings and for each calibration: Calibration data, sample and control data and calibration curve plot.

9. The measured average values in $\%$ represent the Y-values for 1 day (Table 9). Since the values are obtained by automatic interpolation on the calibration curve, these values are expressed as relative concentration factors $\left[F_{R}(1-6)\right]$ of the Reference Material. To make them comparable with the $\mathbf{X}$-values, the following calculation is made (e.g., for Sample 1):

\section{$F_{\mathrm{R}}(1)=$ Average $($ Sample 1$) /\left(\right.$ Predilution Factor $F_{T} 1$ \\ $\times$ Correction Factor $(T) \times 100)$ \\ $=10.9633 /(0.091177 \times 1.0000 \times 100)$ \\ $=1.20242=\underline{\underline{1.2024}}$}

The actual data used are found in Tables 9, 6 and 2, respectively.

The Control Value is calculated in the following way:

\section{Day \#1 = Average (Control)/Relative Concentration in $\%=9.1650 / 9.0169=\underline{\underline{1.0164}}$}

The actual data are from Tables 9 and 5, respectively.

10. The calibration, the dilutions and the measurements of the Samples for 1 day are now completed and the results can be listed as (Table 10):

a. the X-values of the Samples $\left[F_{T} 2(1-6)\right]$ representing the calculated relative concentra- tion values as obtained by dilutions of the Target Material into 6 Samples and

b. the Y-values of the Samples $\left[F_{R}(1-6)\right]$ representing the measured relative concentration values as obtained by interpolation of the signals of the 6 Samples on the calibration curve.

11. The control sample serving as a curve control of the calibration curve is expected to be 1.000 and is found to be 1.0164, which is very good, indicating that the experimental setup has worked satisfactorily.

12. If the Reference Material and the Target Material show true Proportionality, a single straight line should be obtained, if the $\mathbf{Y}$-values are plotted against the $\mathbf{X}$-values. Since the $\mathbf{X}$-values are calculated based on weighing (with 4 decimals), the uncertainty can be regarded as negligible compared to the uncertainty of the interpolated values measured in the immunochemical method (turbidimetry). It is therefore recommended to perform a least squares linear regression analysis, where:

$\mathbf{Y}=\alpha+\beta X$

As seen from Table 11, a zero intercept for the regression is definitely within the confidence interval, permitting a new calculation, in which the regression line is forced through zero (Figure 1, Table 12).

13. According to (3) the slope is equal to the ratio of the concentrations of $\operatorname{lgG}$ in the two materials:

$\boldsymbol{\beta}=\mathrm{C}_{\mathrm{T}} / \mathrm{C}_{\mathrm{R}}$

Since the slope has been calculated from the linear regression analysis, the unknown concentration $\mathbf{C}_{\mathrm{T}}$ of

Table 9 Human IgG - measured values obtained by interpolation.

\begin{tabular}{lccccccr}
\hline & Sample 1 & Sample 2 & Sample 3 & Sample 4 & Sample 5 & Sample 6 & Control \\
\hline $\begin{array}{l}\text { Run \# 1 } \\
\text { Repl. 1, \% }\end{array}$ & 10.9300 & 9.9900 & 8.6700 & 6.3800 & 4.3100 & 2.7800 & 9.2600 \\
$\quad$ Repl. 2, \% & 10.8100 & 9.9000 & 8.6400 & 6.5600 & 4.4000 & 2.7700 & 9.0500 \\
$\quad \begin{array}{l}\text { Mean, \% } \\
\text { Run \# 2 }\end{array}$ & 10.8700 & 9.9450 & 8.6550 & 6.4700 & 4.3550 & 2.7750 & 9.1550 \\
$\quad$ & & & & & & \\
Repl. 1, \% & 11.0000 & 9.9900 & 8.8800 & 6.3800 & 4.1900 & 2.8100 & 9.2700 \\
Repl. 2, \% & 10.9100 & 9.9600 & 8.7600 & 6.4900 & 4.3100 & 2.8200 & 9.0200 \\
$\quad$ Mean, \% & 10.9550 & 9.9750 & 8.8200 & 6.4350 & 4.2500 & 2.8150 & 9.1450 \\
Run \# 3 & & & & & & & \\
$\quad$ Repl. 1, \% & 11.0500 & 10.0200 & 8.8000 & 6.4700 & 4.3600 & 2.7700 & 9.1100 \\
$\quad$ Repl. 2, \% & 11.0800 & 9.9100 & 8.6600 & 6.5500 & 4.4000 & 2.7700 & 9.2800 \\
$\quad$ Mean, \% & 11.0650 & 9.9650 & 8.7300 & 6.2350 & 4.3800 & 2.7700 & 9.1950 \\
Average, \% & 10.9633 & 9.9617 & 8.7350 & 6.4717 & 4.3283 & 2.7867 & 9.1650 \\
\hline
\end{tabular}

Table 10 Human IgG - calculated and measured values of the Samples - day 1.

\begin{tabular}{llllllrr}
\hline Day \#1 & Sample 1 & Sample 2 & Sample 3 & Sample 4 & Sample 5 & Sample 6 & Control \\
\hline X-values & 1.0000 & 0.8984 & 0.7974 & 0.5921 & 0.3977 & 0.2470 \\
Y-values & 1.2024 & 1.0926 & 0.9580 & 0.7098 & 0.4747 & 0.3056 & 1.0164 \\
\hline
\end{tabular}


Table 11 Human Ig G - least squares linear regression - day 1.

\begin{tabular}{lrl}
\hline & & SD \\
\hline Slope & 1.2034 & 0.0109 \\
Intercept & 0.0018 & 0.0077 \\
Slope High & 1.2443 & \\
Intercept Low & -0.0272 & \\
Slope Low & 1.1625 & \\
Intercept High & 0.0308 & \\
\hline
\end{tabular}

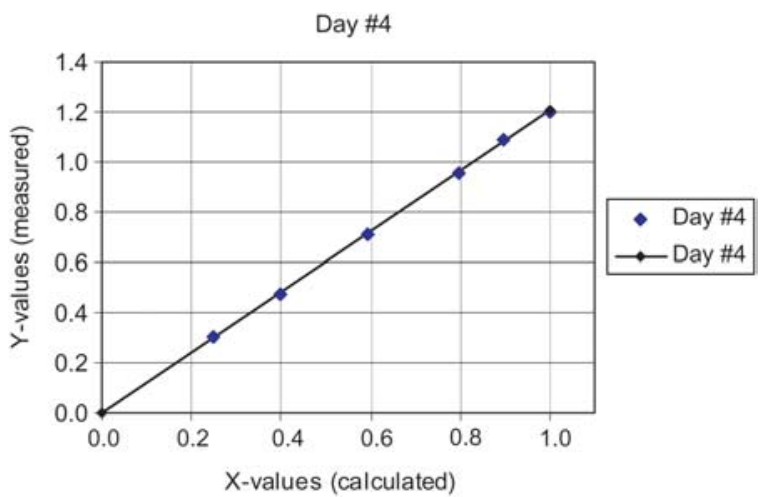

Figure 1 Direct value assignment of human IgG.

The measured relative concentration values of the samples plotted against the calculated relative concentration values of the samples.

Table 12 Human IgG - least squares linear regression forced through zero - day 1.

\begin{tabular}{lll}
\hline & & SD \\
\hline Slope & 1.2057 & 0.0037 \\
Intercept & 0.0000 & \\
Slope High & 1.2183 & \\
Slope Low & 1.1931 & \\
$\mathbf{R}$ & 0.9998 & \\
\hline
\end{tabular}

IgG in the Target Material can now easily be found as:

$\mathbf{C}_{\mathrm{T}}=\mathbf{C}_{\mathrm{R}} \times \boldsymbol{\beta}$

where $\mathbf{C}_{\mathbf{R}}$ is the corrected concentration value of IgG after reconstitution according to Table 1 . So, for 1 day the following concentration of $\operatorname{lgG}$ has been found:

$\mathrm{C}_{\mathrm{T}}=9.68 \mathrm{~g} / \mathrm{L} \times 1.005025 \times 1.2057=\underline{\underline{11.7298 \mathrm{~g} / \mathrm{L}}}$

14. To accomplish a full Direct Value Assignment, the setup for 1 day is carried out a total of 4 times, preferably on 4 different days but at least on 2 different days with a completely new start from reconstitution and dilution every time. It is also mandatory to use the same instrument, the same operator and the same lot numbers of all reagents every time.
15. An example of a full value assignment is shown in Figure 2. The final concentration value for lgG in the Target Material was found to be:

$C T=\underline{11.69 \pm 0.12 \mathrm{~g} / \mathrm{L}}$

with a $\mathbf{C V}=1.1 \%$

16. The IgG concentration in the Reference Material CRM 470 (ERM-DA 470) is stated as $9.68 \mathrm{~g} / \mathrm{L}$ with an uncertainty of $0.10 \mathrm{~g} / \mathrm{L}$.

The IgG concentration in the Target Material was found to be $11.69 \mathrm{~g} / \mathrm{L}$ with an uncertainty of $0.0617 \mathrm{~g} / \mathrm{L}$, as shown in Figure 2.

The total weighted uncertainty is found to be:

$$
\begin{aligned}
& \text { Uncertainty } y_{\text {Total }}=\sqrt{\left[\left(\mathrm{U}_{\mathrm{R}} / \mathrm{C}_{\mathrm{R}}\right)^{2}+\left(\mathrm{U}_{\text {Transfer }} / \mathrm{C}_{\mathrm{T}}\right)^{2}\right]} \times \mathrm{C}_{\mathrm{T}} \\
& \begin{aligned}
\mathrm{U}_{\text {Total }} & =\sqrt{\left[(0.10 / 9.68)^{2}+(0.0617 / 11.69)^{2}\right]} \times 11.69 \\
= & 0.1356 \mathrm{~g} / \mathrm{L}
\end{aligned}
\end{aligned}
$$

From this, it can be seen that $\mathbf{U}_{\text {Total }}$ is mostly influenced by the uncertainty of the Reference Material and very little by the uncertainty of the value transfer.

\section{Acceptance criteria}

After several years of experience, the following general rejection and acceptance criteria for single data points, data series or whole data sets are now used. A very important criterion is visual inspection. By plotting the data from each day in different colors, precision and trueness can easily be detected and separated:

\section{Rejection:}

1. The protocol was not followed.

2. All data points are outside the calibration range.

\section{Acceptance:}

1. The zero intercept is accepted if the numeric value of the intercept $\pm 4 \times$ std. dev. $<0.01$.

2. The mean control value is within $1.0000 \pm 0.05$.

3 . All results are within the overall mean $\pm 3 \times S D$.

\section{Alternative protocol for closed measuring systems}

In closed measuring systems, it is not possible to use the Reference Material as the calibrator and directly assay the Target Material as samples. In these systems, the manufacturer's dedicated calibrator must be used as the calibrator and both the Reference Material and the Target Material assayed as samples. In a closed system, the preparation and dilutions of the Reference and Target Materials are made in essentially the same fashion as discussed above, except that all dilutions must have concentrations within the linear portion of the required calibration curve. This requires predetermination of the approximate con- 


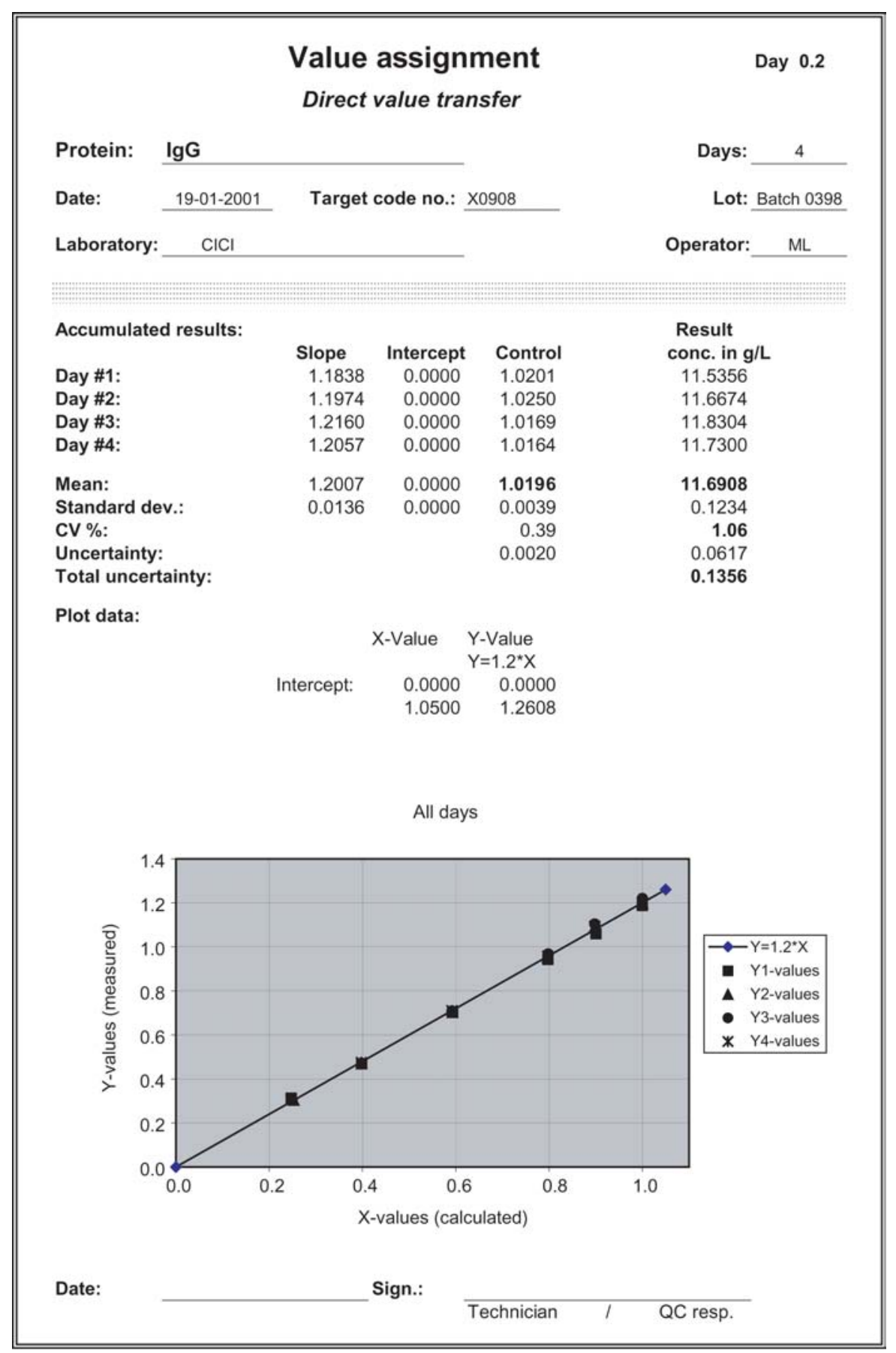

Figure 2 Direct value assignment of human IgG.

Final statistics, plot and results of a full value assignment as they are presented in the Excel spreadsheet.

centrations of the two materials to be compared. The number of assays and replicates is the same as above.

The data in this case will therefore give two regression lines, with the dilutions of each material plotted against the concentrations as determined against the manufacturer's calibration curve. Because the X-values for both materials are calculated based on weighing (with 4 decimals) the uncertainty can be regarded as negligible. Therefore, a least squares linear regression analysis - not a Deming regression - should be used.

If there are no matrix effect problems among the three materials, and the calibration curve has been properly determined, both lines should give zero intercepts within the limits discussed above. If the slopes of the two regression lines are then calculated without an intercept, the ratio of these two slopes will give the ratio of the concentrations of the analyte in the two materials:

$$
\mathbf{C}_{\mathrm{T}}=\mathrm{C}_{\mathrm{R}} \times\left(\boldsymbol{\beta}_{\mathrm{T}} / \boldsymbol{\beta}_{\mathrm{R}}\right)
$$

However, if the two regressions are linear but both show a similar, non-zero intercept, the ratio of their concentrations can be determined, but it should be 


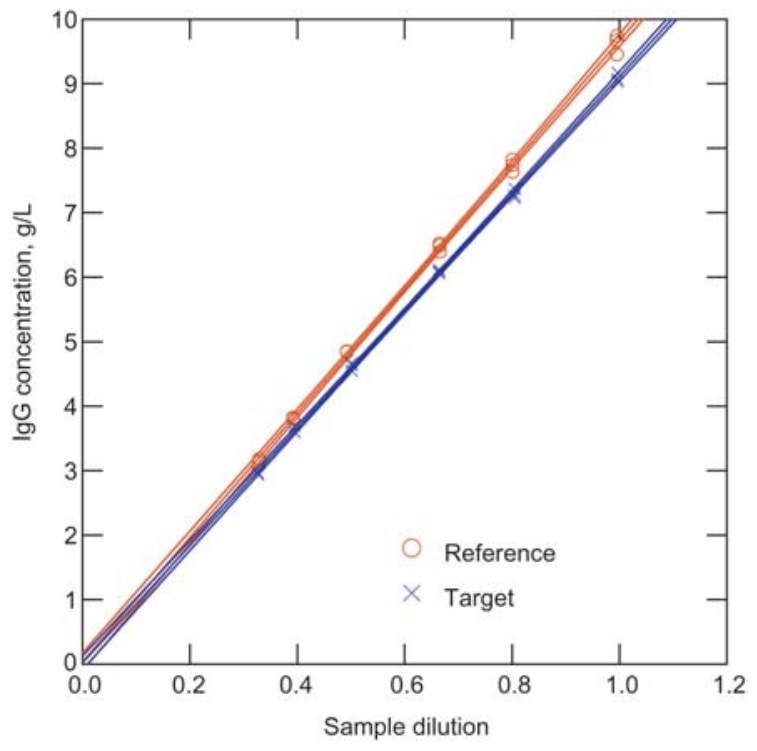

Figure 3 Value assignment of human IgG in closed measuring system.

Table 13 Human Ig G - least squares linear regression - day 1.

\begin{tabular}{lll}
\hline & & SD \\
\hline Slope (Target) & 9.0930 & 0.0597 \\
Intercept (Target) & 0.0223 & 0.0392 \\
Slope (Reference) & 9.6360 & 0.0780 \\
Intercept (Reference) & 0.0370 & 0.0511 \\
\hline
\end{tabular}

Table 14 Human IgG - least squares linear regression forced through zero - day 1.

\begin{tabular}{lll}
\hline & & SD \\
\hline Slope (Target) & 9.1246 & 0.0208 \\
Intercept (Target) & 0.0000 & \\
Slope (Reference) & 9.6886 & 0.0274 \\
Intercept (Reference) & 0.0000 & \\
\hline
\end{tabular}

noted that the manufacturer's calibrator shows a matrix difference, and/or the calibration equation is incorrect. If the same calibration method is to be used for assaying patient samples accurately, it is essential that the reason for the non-zero intercepts be determined and the problem be corrected. Otherwise, the results can be correct at only one point of the calibration curve.

The graph in Figure 3 shows the actual regression lines for 1 day in a recent value assignment in a closed measuring system using CRM 470 as the Reference Material.

In Table 13, the least squares regressions give the values for the Target Material and the Reference Material.

As seen from Table 13, both intercepts are not significantly different from zero, permitting a new calculation, in which the regression lines are forced through zero (Table 14).
The concentration in the Target Material is then calculated as:

\section{$\mathrm{C}_{\mathrm{T}}=(9.1246 / 9.6886) \times 9.68 \mathrm{~g} / \mathrm{L} \times$ Corr. Factor $=9.12 \mathrm{~g} / \mathrm{L}$}

where $9.68 \mathrm{~g} / \mathrm{L}$ is the $\mathrm{IgG}$ concentration assigned to CRM 470 corrected with the reconstitution factor, which in this case was the same for both materials.

\section{Discussion}

When increasing amounts of an antigen is added to a constant amount of the corresponding antibody, a continuous increasing signal (due to the immune complexes) is formed. However, as demonstrated by Heidelberger and Kendall in 1935 (9), this reaction does not form a straight line but a curved curve, which passes through an optimum reflecting the maximum binding capacity of the antibody. This is a unique and special feature of all immunochemical reactions taking place in solution. For immunoturbidimetry and immunonephelometry, the most reliable measurements are obtained on the left-hand side of the immunoprecipitation curve, i.e., in the antibody excess zone.

The value assignment protocol is designed to take advantage of all these conditions. The setup is made in such a way that the reaction curve of the 6 different dilutions of the Reference Material is directly compared to the reaction kinetics of the 6 different dilutions of the Target Material. If they behave in the same way when reacting with the corresponding antibody, i.e., they show the same non-linear reaction kinetics, they are considered to be proportional (show proportionality), and a linear relationship passing through zero can be established when plotting the measured relative concentration values of the samples against the calculated relative concentration values of the same samples. The slope of this regression line will reflect the ratio of the analyte concentration in the two materials.

The exact same principles should be considered, when a patient sample is assayed and interpolated on a calibration curve. Only if the two materials show proportionality can a reliable result be obtained over the calibration interval, i.e., the measuring range.

The reduction of uncertainty in clinical assays is important for several reasons, including in particular the improvement of patient care, both in diagnosing diseases and in following changes due to disease and treatment of the patient. In addition, the comparability of values among assays is necessary for the development of common reference intervals and for comparison of results among various population groups (10).

The same reaction principles can be studied in a closed measuring system provided the manufacturer's calibrator behave in the same proportional way as the Reference Material and the Target Material. 
The more theoretical principles of the value assignment are outlined in our first paper (3). In this paper, a more practical approach has been presented enabling manufacturers and others to conduct value assignment in the most optimal way leading to more reliable protein values of the calibrator and thus to better and more comparable protein values for the patient.

As discussed above, persisting among-manufacturer variation in protein assays may result from several factors, but the only one of these that can be readily addressed is the assignment of values to the materials used for calibrators and controls. The protocol presented here is designed to perform the assignment of values to both master calibrators and commercial, or "working," calibrators with as little uncertainty as is practically possible.

The protocol requires little if any effort over and above methods in common use by manufacturers and has been shown to result in a significant reduction in uncertainty of assigned values. We strongly encourage its use for all serum protein value assignments.

\section{References}

1. Baudner S, Bienvenu J, Blirup-Jensen S, Carlström A, Johnson AM, Ward AM, et al. The certification of a matrix reference material for immunochemical measurement of 14 human serum proteins, CRM 470. EUR 15243 EN, 1993:1-186.

2. Svendsen PJ, Blirup-Jensen S, Johnson AM. Certification campaign (chapter 7). Protocols for value transfer (chapter 12.4). In: Baudner S, Bienvenu J, Blirup-Jensen S, Carlström A, Johnson AM, Ward AM, et al., editors. The cer- tification of a matrix reference material for immunochemical measurement of 14 human serum proteins, CRM 470. EUR 15243 EN, 1993:1-186.

3. Blirup-Jensen $S$, Johnson AM, Larsen M. Protein standardization IV: value transfer. Procedure for the assignment of serum protein values from a reference preparation to a target material. Clin Chem Lab Med 2001;39:1110-22.

4. Johnson AM, Whicher JT. Effect of certified reference material 470 (CRM 470) on national quality assurance programs for serum proteins in Europe. Clin Chem Lab Med 2001;39:1123-8.

5. Johnson AM, Whicher JT, Ledue TB, Carlström A, Itoh $\mathrm{Y}$, Petersen PH. Effect of a new international reference preparation for proteins in human serum (Certified Reference Material 470) on results of the College of American Pathologists surveys for plasma proteins. Arch Pathol Lab Med 2000;124:1496-501.

6. Ledue TB, Johnson AM, Cohen LA, Ritchie RF. Evaluation of proficiency survey results for serum immunoglobulins following the introduction of a new international reference material for human serum proteins. Clin Chem 1998;44:878-9.

7. Ledue TB, Johnson AM. Commutability of serum protein values: persisting bias among manufacturers using values assigned from the certified reference material 470 (CRM 470) in the United States. Clin Chem Lab Med 2001;39:1129-33.

8. Blirup-Jensen S. Protein standardization III. Method optimization. Basic principles for quantitative determination of human serum proteins on automated instruments based on turbidimetry or nephelometry. Clin Chem Lab Med 2001;39:1098-109.

9. Heidelberger $M$, Kendall FE. A quantitative theory of the precipitin reaction. J Exp Med 1935;62:697.

10. Johnson AM, Hyltoft Petersen P, Whicher JT, Carlström A, MacLennan S. Reference intervals for serum proteins: similarities and differences between adult Caucasian and Asian Indian males in Yorkshire, UK. Clin Chem Lab Med 2004;42:792-9. 